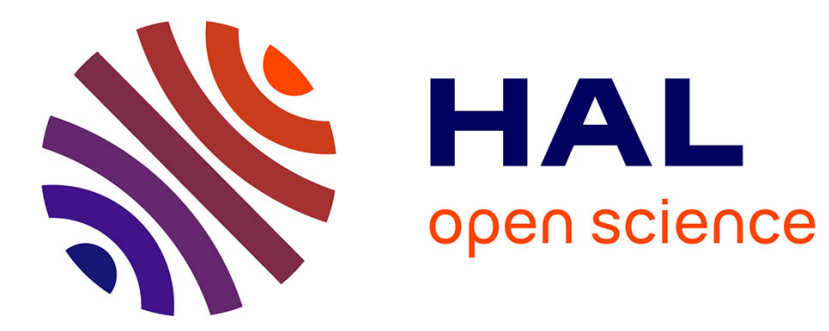

\title{
Commémoration des origines de la foresterie turque
}

Michel de Galbert

\section{To cite this version:}

Michel de Galbert. Commémoration des origines de la foresterie turque. Revue forestière française, 2013, 65 (6), 10.4267/2042/53996 . hal-03447494

\section{HAL Id: hal-03447494 \\ https://hal.science/hal-03447494}

Submitted on 24 Nov 2021

HAL is a multi-disciplinary open access archive for the deposit and dissemination of scientific research documents, whether they are published or not. The documents may come from teaching and research institutions in France or abroad, or from public or private research centers.
L'archive ouverte pluridisciplinaire $\mathbf{H A L}$, est destinée au dépôt et à la diffusion de documents scientifiques de niveau recherche, publiés ou non, émanant des établissements d'enseignement et de recherche français ou étrangers, des laboratoires publics ou privés. 


\title{
HISTOIRE ET TERAITOIRES
}

\section{COMMÉMORATION DES ORIGINES \\ DE LA FORESTERIE TURQUE}

\author{
Michel de Galbert
}

\section{LA CONTRIBUTION DES FORESTIERS FRANÇAIS À LA NAISSANCE DE LA FORESTERIE TURQUE : UN PARTENARIAT FÉCOND}

La faculté forestière d'Istanbul a organisé les 18 et 19 novembre 2013 un colloque sur ses origines. Or plusieurs forestiers français, notamment Louis Tassy, ont contribué au démarrage de ses activités entre 1857 et 1865 .

À la demande de Bernard Roman-Amat, directeur du centre de Nancy d'AgroParisTech, c'est Michel de Galbert, membre du Conseil général de l'alimentation, de l'agriculture et des espaces ruraux, qui a représenté AgroParisTech à ce colloque réunissant 200 forestiers et étudiants turcs issus de plusieurs universités de tout le pays. Après une première cérémonie récompensant d'anciens professeurs de foresterie, l'ouverture du colloque a permis à une représentante de l'ambassade de France d'introduire les enjeux de développement durable représentés par la foresterie. Michel de Galbert a ensuite présenté en anglais un exposé dont les éléments suivent.

Ce colloque fut l'occasion d'échanges fructueux avec les forestiers turcs, et semble avoir montré une volonté de reprise de contact entre la faculté forestière d'Istanbul et AgroParisTech, en vue de collaborations ultérieures.

\section{LE CONTEXTE}

L'immense empire ottoman comprenait des vastes forêts, qui, comme les terres, appartenaient à l'État. Mais à l'origine, après la conquête territoriale, l'administration ne se préoccupait pas de gestion, et les populations pouvaient user des forêts sans limitation. Seules quelques mesures avaient été prises pour satisfaire les besoins en bois de l'armée, de la marine et du gouvernement. Pendant l'ère du Tanzimat, qui commença en 1839, des réformes furent lancées pour valoriser les ressources et résoudre la crise financière. Une taxe fut alors créée sur les produits forestiers, ainsi qu'un service forestier destiné à la collecter. Mais les fonctionnaires chargés de cette collecte n'avaient aucune formation forestière, les résultats furent décevants et le service rapidement supprimé.

En 1856, à la fin de la guerre de Crimée, le gouvernement souhaitait lancer des réformes radicales dans tous les domaines et, dans cette perspective, un conseil des Travaux publics fut créé. 


\section{DES FORESTIERS FRANÇAIS EN TURQUIE, POUR UNE PREMIÈRE MAIS BRÈVE PÉRIODE}

Ce conseil fut conçu pour donner son avis sur toutes les questions concernant la prospérité économique du pays: infrastructure, mines, organisation forestière. Ce conseil était l'une des plus hautes instances de décision de l'empire ottoman. Deux inspecteurs forestiers, Tassy et Sthème, l'ont rejoint. Pour accomplir leur mission forestière, ils furent accompagnés par huit autres forestiers.

Cette première mission prit fin en 1862. Une seconde mission, avec Godchaux, Montrichard et Galmiche, commença en 1870 et prit fin en 1873. Toutes ces missions furent demandées, renouvelées et financées par le Gouvernement turc.

\section{LES PRINCIPALES CONTRIBUTIONS DE FORESTIERS FRANÇAIS}

Une première école forestière fut créée à Istanbul en 1857, préludant la fondation de l'université forestière d'Istanbul. Neuf étudiants passèrent les examens de la première promotion auxquels assistait le Grand Vizir. Ce lancement ne fut pas simple, les livres étant rares et la barrière de la langue bien réelle. Après cette formation initiale, les jeunes forestiers furent envoyés immédiatement sur le terrain pour reconnaître les forêts, les inventorier et rédiger des plans d'aménagement.

Un projet de Code forestier fut lancé, et les premiers services forestiers furent mis en place, visant à donner un statut à la propriété forestière. Le Code forestier fut adopté en 1870. Toutes les forêts appartenaient au sultan. Aucune taxe n'était prélevée sur la population; seule une taxe de 10 à $20 \%$ était prélevée sur les produits d'exportation, avec pour inconvénient de favoriser l'exportation des plus beaux produits.

Une carte des forêts turques à l'échelle de 1/500000 fut dressée, carte hélas disparue aujourd'hui. L'aménagement durable des forêts les plus riches se mit en place dans l'Empire. Des commissions furent installées pour organiser les appels d'offres préalables aux exploitations. Les forestiers français reconnurent 40000 ha à Smyrne, des forêts à Caramania, 250000 ha dans le Mont-Ida (Godchaux), des forêts de Pin laricio en Bulgarie et en Albanie (Montrichard), des forêts de Chêne pour la production de tonneaux en Bosnie (Simon) et en Thessalie (Chervau). Les forestiers limitaient les transgressions et créaient des voies de transport des grumes. En Bosnie, l'exportation de tonneaux vers la France fut envisagée.

\section{DES VOYAGES PITTORESQUES}

La Thessalie n'était pas sûre, et de ce fait Chervau fut accompagné par une escorte de huit gardes!

Galmiche décrit les forêts de l'Anti-Taurus qui couvrent 500 ooo ha comme une "mer d'arbres » le long de la mer Noire, et en observa les principales espèces: Chêne, Hêtre, Sapin, Pin noir (avec un problème de disparition car l'écorce des jeunes pins était très appréciée pour la consommation humaine !).

Les villages étaient environnés de buissons ; les forêts étaient surexploitées près des habitations. Plus loin des villages se trouvaient les forêts jardinées, laissant place à des déserts. Les routes étaient de simples chemins de crête pour les bœufs. Les maisons étaient en bois, comme les clôtures autour des champs et des pâtures. Les voyageurs étaient chaleureusement accueillis, parfois dans une construction légère dans le centre du village ou dans la mosquée. 


\section{TASSY : UNE PERSONNALITÉ ATTACHANTE}

Louis Tassy (1816-1895) était un chercheur avide de contribuer à la connaissance scientifique, en particulier pour l'aménagement forestier, acceptant la controverse ${ }^{(1)}$. Il enseigna à l'Institut national agronomique créé à Versailles en 1848. Louis Tassy fut un grand éducateur, soucieux d'amener ses élèves à une bonne réflexion plutôt qu'à accumuler des connaissances. Indépendant, attiré par les réformes et les idées nouvelles, il proposa au moment de son départ à la retraite une importante réforme de l'administration forestière. Louis Tassy était cultivé, et sa personnalité était empreinte de charme et de tact.

\section{UN BON DÉBUT, POUR DES RELATIONS FORTES ENTRE LES FORESTIERS DES DEUX PAYS}

Les forêts turques et françaises ont beaucoup de points communs : des écosystèmes méditerranéens et montagneux (Pin, Chêne, Hêtre, Cèdre, Sapin, Charme) sur 10 millions d'hectares en Turquie, et près de 6 millions en France (Pin maritime exclus).

Le partenariat noué par Tassy et ses compagnons est un lien fort dans la longue chaîne de relations amicales qui lie nos pays depuis Soliman le Magnifique et François Ier. Souhaitons que ce partenariat soit renouvelé et perdure encore longtemps!

\section{Michel de GALBERT}

Ingénieur général des ponts, des eaux et des forêts CONSEIL GÉNÉRAL DE L'ALIMENTATION,

DE L'AGRICULTURE, ET DES ESPACES RURAUX

251 rue de Vaugirard

F-75732 PARIS CEDEX 15

(michel.de-galbert@agriculture.gouv.fr)

\section{COmmÉmORATION DES ORIGINES DE LR FORESTERIE TUROUE [Résumé]}

La France a contribué à la naissance de la foresterie en Turquie. Le colloque de novembre 2013 organisé à l'université forestière d'Istanbul a été l'occasion d'une communication, ici présentée, de Michel de Galbert au nom d'AgroParisTech.

\section{COMMEMORATION OF THE INCEPTION OF FORESTRY IN TURKEY [Abstract]}

France contributed to the inception of forestry in Turkey. The symposium organised in November 2013 at the Forestry University of Istanbul was the occasion for Michel de Galbert, on behalf of AgroParisTech, to make the presentation described.

\footnotetext{
(1) Il fut aussi vérificateur général des aménagements forestiers à l'administration des forêts de 1868 à 1875 et eut des positions aujourd'hui critiquables sur les sacrifices d'exploitabilité à ne pas hésiter à faire, disait-il, pour aménager les forêts domaniales en futaie régulière.
} 